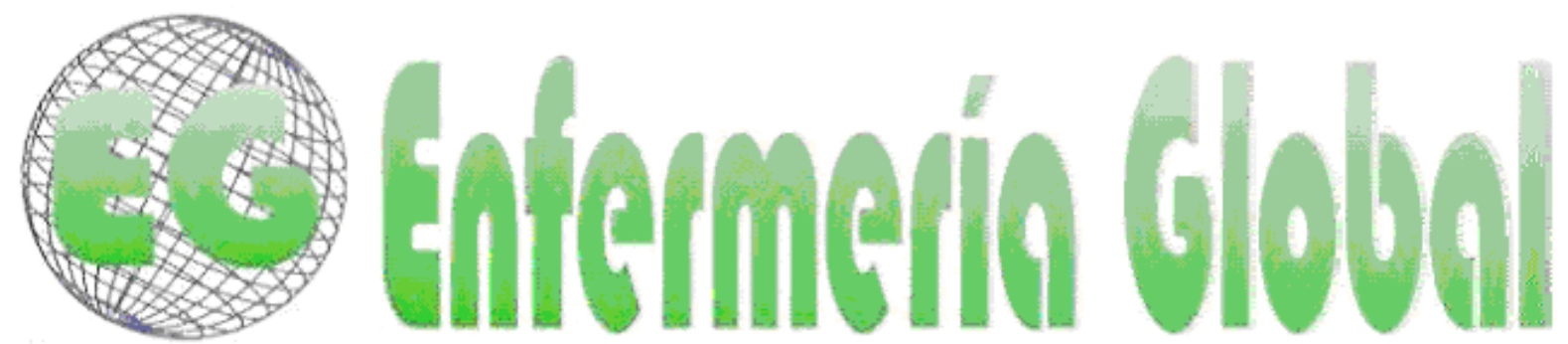

\title{
ENSAYOS
}

\section{Evidencia del Arte en Enfermería}

Evidence of the Art in Nursing

\section{*Contreras Ibacache, Víctor}

\author{
*Enfermero. Candidato a Magíster en Enfermería. Pontificia Universidad Católica de Chile E-mail: \\ vecontre@uc.cl .
}

Palabras clave: Arte; Enfermería; Bases del Conocimiento; Instrucción para Enfermeras.

Keywords: Art; nursing; Knowledge Bases; Instruction for nurses.

\section{RESUMEN}

En este artículo se reflexiona sobre la evidencia del arte de cuidar en enfermería. La raíz de la pregunta surge a través del patrón de conocimiento estético (arte), el cual lo comunicamos de diferentes formas, artículos en revistas, experiencias educativas, la crítica de artículos científicos entre otras. Los argumentos que sustentan esta reflexión están enlazados con la formalidad de comunicar, las maneras de explorar el conocimiento estético y finalmente la influencia del artista, como hacedor y critico del arte de cuidar. Es un desafío reconocer nuestro quehacer como un arte, dar a conocerlo e indagar acerca de éste, el cual puede estar en el foco de la investigación con metodología cualitativa, como una herramienta para rescatar el lenguaje comprensivo que nos identifica

\section{ABSTRACT}

In this article I try to examine the evidence of the art of caring in nursing. The following question arises through the pattern of aesthetic knowledge (art), which we could communicate in different ways such as journal articles, educational experiences, critique of scientific articles and more. The arguments behind this thinking are linked to the formality of communication, the ways to explore aesthetic knowledge and finally the influence of the artist as maker and critic of the art of nursing care. We have a challenge to recognize our work as an art, to make it known and inquire about it, which can be the focus of qualitative research methodology as a tool for collecting comprehensive language that identifies us.

\section{INTRODUCCIÓN}

Históricamente las manifestaciones artísticas han sido expresiones de la cultura en la cual las personas están inmersas. Son actos mediante los cuales, usando la materia se imita o se expresa las emociones de la persona, lo invisible ${ }^{(1)}$. Esta creación, obra, producto del ser humano, busca plasmar las ideas, emociones y/o sentimientos, 
convertidas en expresiones que obedecen a los propios patrones de belleza y estética, con la finalidad de entregar una visón del mundo, estética o comunicativa ${ }^{(1,2)}$.

Para Chinn \& Kramer $^{(3)}$ el conocimiento estético (arte) en enfermería es un profundo reconocimiento del significado de una situación, en la cual deben convocarse los recursos creativos internos que transforman la experiencia. Éste se hace visible a través de la acción, conductas, actitudes, narrativa e interacciones de un(a) enfermero(a) en relación con otros.

Así también, el acto artístico de cuidar (expresiones verbales y no verbales) es una relación única entre el (la) enfermero(a) y la persona (individuo y/o comunidades), como forma de expresión transformativa de la realidad que simboliza una experiencia ${ }^{(3)}$, el cual puede o no comunicarse a través de lo que conocemos hoy como evidencia estética ${ }^{(4)}$.

Siguiendo la idea propuesta por Porter ${ }^{(4)}$, es problemático atribuir a las formas pintura, escultura, cine, danza, y otras "obras de arte", como la única vía de comunicación de la evidencia estética. A partir de esta perspectiva la pregunta que surge para esta reflexión es: ¿Cómo evidenciamos el arte de enfermería?.

Para tratar de responder esta pregunta, trataremos de buscar los argumentos que sustentan la formalidad de comunicar, la maneras de explorar el conocimiento estético y finalmente la influencia del artista, como hacedor y critico del arte de cuidar.

\section{FORMALIDAD DEL COMUNICAR EL ARTE}

Al iniciar la reflexión sobre el arte, vale la pena destacar que el cuidado de enfermería profesional, se distingue del cuidado innato de los seres humanos, porque éste trata de establecer un proceso intencional altruista de querer ayudar a los otros en sus procesos de salud y enfermedad, muerte y renacimiento, fundando y guiando este cuidado en conocimiento teórico ${ }^{(5)}$.

A partir de este conocimiento teórico se pueden establecer diferentes formas de dar a conocer los que el cuidado profesional realiza día a día, lo que conocemos como evidencia científica en enfermería ${ }^{(5)}$. Es aceptada por la comunidad científica de enfermería una vía formal de comunicación sobre los cuidados enfermeros, conocida como revistas científicas, las cuales cuentan con un comité editorial, en formato impreso y/o electrónico.

Dentro de esta vía formal, se pueden encontrar diversos y numerosos textos relacionados con estudios guiados bajo la metodología cuantitativa y/o cualitativa, así también reflexiones personales, cartas editoriales, comentarios académicos, entre otros.

Del mismo modo, desde la perspectiva del curador de arte, E. Ramírez ${ }^{(6)}$ propone que las revistas pueden ser un proceso de comunicación, una red, un proceso creativo colectivo, un espacio de experimentación (tipo)gráfica, un formato práctico y de amplio alcance para acumular y difundir proyectos, un proceso de (re)producción, un vehículo ideológico y parte de la institución crítica.

Como proceso creativo tipográfico y narrativo las revistas científicas serían piezas del arte de enfermería; así como otras publicaciones de asociaciones de enfermeros 
(sitios web sobre teoría, filosofía, modelos de enfermería) aportan a la concienciación global de la enfermería ${ }^{(5,7)}$.

Las formas narrativas (investigaciones, reflexiones, cartas editoriales, etc.) comunicadas en las revistas científicas, son en sí mismas un acto artístico narrativo del arte de enfermería dadas a conocer mediante la comunicación escrita de la experiencia de la relación con otros en el ámbito del cuidado profesional. Las cuales pueden ser aceptadas, criticadas y (re)utilizada por la comunidad de enfermería para exponer lo propio de la profesión y ciencia del cuidado.

\section{OTRO ENFOQUE DE COMUNICAR EL ARTE DE CUIDAR}

En la búsqueda de comunicaciones científicas referentes al arte de enfermería, sorprenden las propuestas de comunicar nuestro quehacer que fortalecen la idea de esta reflexión.

De acuerdo con C. Delaney ${ }^{(8)}$, quien publica una experiencia pedagógica realizada con sus alumnos de enfermería en el Museo de Arte de su ciudad, cuyo objeto fue explorar el conocimiento estético en enfermería. Se puede evidenciar la reflexión de los alumnos del patrón estético por medio de una experiencia en el Museo de Arte.

Se puede deducir de la experiencia de Delaney ${ }^{(8)}$ que la figura del curador de arte, es símil a la del (la) enfermero(a) pues éste reconoce trabajo personal del artista en su contexto, ayudando a contextualizar la expresión plasmada en la obra a observar. Lo cual en el escenario de enfermería podría traducirse como percepción del cuidado y del cuidador, mirado desde la perspectiva de patrón estético ligado íntimamente a las acciones del cuidado.

De la misma manera, cabe reconocer que las obras del cuidado de enfermería son únicas, verdaderas e individuales, son un aporte innovador y actual de la realidad de las personas.

Loable trabajo de Delaney con sus alumnos ${ }^{(8)}$ pues integrar el patrón de conocimiento estético desde la formación es una forma de comunicar nuestro arte tarea de muchos de nosotros como mentores, guías, profesores, maestros.

Otra experiencia que enriquece esta reflexión es la propuesta por Northington, Wilkerson, Fisher y Schenk ${ }^{(9)}$, los cuales describieron la implementación de una reflexión del patrón estético de enfermería en el ambiente pediátrico de un programa de formación en enfermería, a través de los aportes de literatura "no" científica (novelas, cuentos, entre otros) y material audiovisual (películas comerciales).

De acuerdo con Northington, Wilkerson, Fisher y Schenk ${ }^{(9)}$, podemos decir que el uso de herramientas literarias "no" científicas y audiovisuales - consideradas piezas de arte - cuyo contenido tenía relación con la experiencia de vivir con enfermedad, abuso, discapacidad y/o muerte de una persona y la perspectiva de la familia, podría favorecer la reflexión, por medio del patrón estético, de la experiencias vividas en el clínica por los estudiantes de enfermería en el ambiente pediátrico.

Nuevamente, a la luz del proceso de sensibilización, comprensión e integración de patrón estético en la experiencia vivida, puede ser un camino para comunicar nuestro arte. 
Por lo tanto, para los que cuidamos a otros, este tipo de experiencias aprendizaje aportan a comprender el comportamiento humano y sus respuestas, que a menudo es difícil, debido a las variadas expresiones de las personas en relación a su salud y enfermedad ${ }^{(9,10)}$

Tareas como las propuestas por Northington et al. ${ }^{(9)}$ y Delaney ${ }^{(8)}$ permiten la integración y reflexión sobre el arte de cuidar en la cual se empodera al estudiante a pensar acerca de sus reacciones en los escenarios posibles antes de tener que intervenir en un entorno real.

En complemento con lo anterior, decir que los ambientes de simulación clínica podrían llegar a integrar los elementos del arte de cuidar, convirtiéndose en una forma de evidencia del patrón de conocimiento estético, a través de la puesta en escena de situaciones que vivimos a diario, re-mirando lo que hacemos.

Por otro lado, G Lindsay ${ }^{(11)}$ publicó una breve comunicación acerca de cómo leer artículos de revistas relacionadas con la práctica de enfermería. De acuerdo con la autora, una forma de reconstruir y reconocer los significados de un artículo es leer críticamente éste, rescatando la evidencia que dio soporte a las interpretaciones, el juicio, la crítica o el debate propuesto por su autor.

En pocas palabras, desde la perspectiva de Lindsay ${ }^{(11)}$, se reafirma la idea del "crítico de arte en enfermería" ya que, el leer un artículo es hacer una valoración estética sobre la obra de arte del artista, que inicialmente es una crítica personal y subjetiva, muy humana, para luego valorar la expresión artística según su contexto o evolución, aportando datos empíricos y contrastables ${ }^{(12,13)}$.

Por lo tanto, podríamos proponer que el juicio, la evidencia, la forma y la presentación, son componentes de la crítica que hace un curador de arte al igual que un enfermero con respecto a su práctica, usando diferentes guías para levantar un argumento para aceptar o rechazar una obra de arte.

\section{LA EXPRESIÓN DE LA PERSONA (ENFERMERA/O), COMUNICACIÓN DEL PROPIO ARTISTA}

Primeramente, de acuerdo con la perspectiva de Jean Watson la relación de cuidado humano esta dada como una relación transpersonal (14), unión entre el (la) enfermero(a) y la persona (individuo y/o comunidades), como acto artístico de la expresión de cuidar único y personal ${ }^{(3,5)}$.

A mi modo de ver, la relación transpersonal está basada en la experiencia vivida, las creencias y la autoexploración, las cuales ayudan a los(as) enfermeros(as) a ver e integrar el todo en el acto de cuidar ${ }^{(10)}$.

Al mismo tiempo, el arte es una selectiva recreación de la realidad la cual ofrece otra vía de desarrollo de comprender los aspectos profundos de los seres humanos en el trabajo de enfermería ${ }^{(16)}$

Por lo cual podemos llegar a decir, creer y confirmar día a día en nuestro quehacer que cada acto único y personal es en sí mismo una pieza de arte de nuestra profesión, el cual es comunicado a otros en el mismo acto de cuidar, ejemplificando, cuando saludo a una persona en el momento de entrar a la habitación del hospital, el 
acto mismo de saludar con la intención de cuidar es una pieza de arte en la cual dos personas se encuentran.

Sin embargo, hoy día es un desafío hablar de arte en enfermería, y concordamos con Dra. M. S. Rivera quien también le llama "...la atención como estamos comunicando nuestro conocimiento estético. De cierto modo, muy primario aún, creo que muchas veces no sabemos cómo hacerlo, nos quedamos en lo "científico".... Bueno...ahí está el gran aporte de la investigación comprensiva de las experiencias vividas. Ahí está el aporte de la investigación cualitativa, que proporciona un marco teórico y filosófico que valorando la experiencia humana, ... formas de estudiarla y acoger su esencia y comunicarla en lenguaje..." (M.S. Rivera, comunicación personal, 19 de Junio 2012).

Por lo cual reafirmamos la idea de que la experiencia vivida en el cuidado sería un acto artístico en sí mismo, el cual nos insta a comunicar consciente o inconscientemente lo vivenciado, a reconocer que "... arte de la enfermería es difícil de explicar y conceptualizar..." (K.O -solicitó resguardar su identidad-, comunicación personal, 20 de Junio 2012) contamos con instancias para darlo a conocer. $Y$ al mismo tiempo, lo comunicamos en el momento que nos relacionamos transpersonalmente, siendo capaces de "... comprender y adaptarse a una variedad de ambientes... utilizando habilidades de comunicación una manera apropiada en cada caso, en el cual el foco es el paciente de manera integral" (K.O, comunicación personal, 20 de Junio 2012).

Finalmente, podemos agregar que hoy en cada momento de nuestro quehacer (al cuidar, educar en cuidados, investigar y comunicar científicamente lo que hacemos), estamos mostrando nuestro arte, el cual tiene un potencial enorme para comprender la experiencia humana en su totalidad.

\section{CONSIDERACIONES FINALES}

La forma comunicación de la evidencia estética en enfermería, no es necesariamente a través de la pintura, escultura y otras "obras de arte".

Con cada momento de cuidado los(as) enfermeros(as) podemos hacer visible nuestro arte y al mismo tiempo podemos plasmarlo en acciones que son apreciables en sí mismas como obras de arte (desde comunicaciones científicas hasta una simple mirada a la persona que se cuida).

La formación profesional y las intervenciones innovadoras como la simulación, son nuevos desafíos para ayudar a la comprensión y la comunicación del patrón estético en enfermería.

Así mismo, somos nuestros propios curadores de arte, quienes tratamos de comprender el arte de la enfermería, criticando nuestra forma de cuidar y de cierto modo lo elevamos a un acto único que se da en el contexto de la relación con otro(s). Sin embargo, es un desafío reconocer nuestro quehacer como un arte, dar a conocerlo e indagar acerca de éste, el cual puede estar en el foco de la investigación con metodología cualitativa, como una herramienta para rescatar el lenguaje comprensivo que nos identifica. 
Como consecuencia, los artistas de nuestro quehacer somos nosotros, jugando un rol protagónico en el momento de cuidar, siendo mentores y aprendices, capaces de desarrollar y comunicar el arte de la enfermería en el mundo actual.

\section{REFERENCIAS}

1. Velandia LI. Historia del arte [Internet]; 2010. [cited 2012 Jun 4]. Available from: http://www.monografias.com/trabajos13/histarte/histarte.shtml

2. Wikipedia a. Historia del arte. arte [Internet]; 2012. [cited 2012 Jun 6]. Available from: http://es.wikipedia.org/wiki/Historia_del_arte

3. Chinn P, Kramer M. Theory and Nursing integrated knowledge develpoment. 5ta. ed. Missouri: Mosby; 1999. Chapter 1, Nursing's fundamental patterns of knowing: $p$. $1-15$.

4. Porter S. Fundamental patterns of knowing in nursing: the challenge of evidencebased practice. Ans Adv Nurs Sci. 2010 Jan-Mar, 33(1): 3-14.

5. Rivera S. El arte de cuidar en Enfermería. Horiz Enferm. 2004, 15: 11-22.

6. Ramírez E. La Publicación y su Campo Expandido: el caso documenta 12 magazine. Arte y Crítica. [Internet]; 2008. [cited 2012 Jun 4]. Available from: http://www.arteycritica.org/index.php?option=com_content\&task=view\&id=383\&ltemid $=27$

7. Alligood MR. Modelos y teorías en enfermería. 7th ed. Ámsterdam: Elsevier; 2011. Chapter 37, Estado actual y ciencia de la teoría enfermera; p. 754-761.

8. Delaney C. A trip to the Art Museum as a pedagogical approach to the teachinglearning of nursing aesthetics with RN to BSN students. J Nurs Educ. 2006, 45 (3): 143-144.

9. Northington L, Wilkerson R, Fisher W, Schenk L. Enhancing nursing student's clinical experiences using aesthetics. J Prof Nurs. 2005; 21 (1): 66-71.

10. Mitchell G, Cody W. Ambiguous opportunity: toiling for truth of nursing art and science. Nurs Sci Q. 2002; 15 (1): 71-79.

11. Lindsay G. Reading journals: Recovery and Reconstruction of meaning for nursing praxis. J Nurs Educ. 2006; 45 (3): 143.

12. Ospina M. Procesos del Arte en Colombia [Internet]. Bogota: Instituto Colombiano de Cultura, Subdirección de Comunicaciones Culturales, División de Publicaciones; 1978. Chapter 32, Encuesta: "La crítica en Colombia" 1957; [cited 2012 May 31]; Available

http://www.banrepcultural.org/blaavirtual/todaslasartes/procesos/cap32.htm

from:

13. Wikipedia b Art criticism. [Internet]; 2012. [cited 2012 Jun 9]. Available from: http://en.wikipedia.org/wiki/Art_criticism

14. Jesse D E. Modelos y teorías en enfermería. 7ma ed. Ámsterdam: Elsevier; 2011. Chapter 7, Filosofía y teoría del cuidado transpersonal de Watson; p. 91-112.

15. Watson J, Biley F, Biley A. Aesthetics, postmodern nursing, complementary therapies and more: an internet dialogue. Complement Ther Nurs Midwifery. 2002; 8: 81-83.

ISSN 1695-6141

๑ COPYRIGHT Servicio de Publicaciones - Universidad de Murcia 